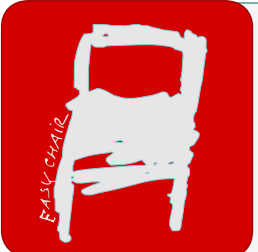

EPiC Series in Health Sciences

EPIC

Health

Volume 4, 2020, Pages 138-143

Sciences

CAOS 2020. The 20th Annual Meeting of the International Society for Computer Assisted Orthopaedic Surgery

\title{
The Incidence of Soft Tissue Releases in Robotic-Assisted Total Knee Arthroplasty Technology
}

\author{
Bertrand Kaper ${ }^{1,2}$ \\ 1 Orthopaedic Specialists of Scottsdale \\ ${ }^{2}$ Scottsdale, AZ USA \\ bkaper@drkaper.com
}

\begin{abstract}
The incidence of formal soft tissues releases required in TKA surgery performed utilizing the NAVIO robotic-assisted (RA)-TKA technique was assessed and compared to a historical cohort of conventional, manually instrumented $(\mathrm{Cl})$ TKA's.
\end{abstract}

\section{Introduction}

Achieving a well-balanced knee has long been recognized as one of the most important goals in total knee arthroplasty (TKA $)^{1}$. Soft tissue balancing is often cited as the "art" of the surgical technique of TKA. Extensive research has been done to guide and optimize the technique of soft tissue releases to balance the knee intra-operatively ${ }^{2,3}$.

With the introduction of robotic-assisted (RA) TKA, planning for bone resections can be customized in the planning stages generating an individualized case plan, based upon a dynamic 3-D model. A hybrid technique of measured resection and gap balancing can thereby be applied, prior to bony resection, to reduce the intra-operative need for soft tissue release.

The goal of this study was to document the incidence of formal soft tissues releases in TKA surgery performed utilizing the NAVIO RA-TKA technique. This incidence was then compared to a historical cohort of conventional, manually instrumented (CI) TKA's. 


\section{Methods}

Starting in August 2019, a longitudinal data collection series was initiated in a singlesurgeon total joint arthroplasty registry. One-hundred and twenty patients, undergoing total knee arthroplasty with the NAVIO 7.0 RA-TKA technique, were enrolled.

A historical cohort of one-hundred patients who had undergone CI-TKA were identified and matched to the RA-TKA group.

For the CI-TKA patient cohort, the surgical technique followed a measured resection protocol as outlined in the manufacturer's technique guide. This included: intramedullary referencing for a five-degree valgus distal femoral osteotomy, with the goal to resect $9.5 \mathrm{~mm}$ medially and $7 \mathrm{~mm}$ laterally; posterior referencing for femoral component sizing; femoral rotation based on the trans-epicondylar axis; extra-medullary tibial referencing for the proximal tibial osteotomy, with the goal to resect $9.5 \mathrm{~mm}$ medially and $12 \mathrm{~mm}$ laterally; and a target of five degrees of posterior tibial slope. Soft tissue balancing was performed following bony resection to create a balanced rectangular extension and flexion spaces with composite implant thickness of $19 \mathrm{~mm}$. Using either a Z-retractor or posterior cruciate ligament (PC) Hohmann retractor to achieve joint distraction, manual varus and valgus stress was applied with final components in place to assess soft tissue tautness or laxity. If imbalance was detected, a formal soft tissue was performed according to the individual circumstances.

For the RA-TKA patient cohort, the planning tools embedded in the NAVIO 7.0 technique were utilized- surface mapping the distal femur and proximal tibia was performed to generate an "imageless", static 3-dimensional model of the distal femur and proximal tibia. A measured resection TKA model, as outlined by the manufacturer's stated surgical technique, was then generated based solely on bone resection depths. Again, using a Zretractor or posterior cruciate ligament (PC) Hohmann retractor to achieve joint distraction, soft tissue laxity assessments were then recorded through a 0-90 "stressed" range of motion- during which both the medial and lateral compartments were subjected to tensile, distractive forces. Adjustments were then made to the plan to achieve a balanced knee ( $<3 \mathrm{~mm}$ of laxity) both medially and laterally. If, through the predictive model, adjustments in component position could not bring soft tissue balance to within $<3 \mathrm{~mm}$, a formal soft tissue release was performed after component placement.

In addition to achieving soft tissue balance, the surgical goal for both patient cohorts was to correct limb alignment, allowing for slight under-correction of either the pre-operative varus or valgus deformity. 
Baseline demographic information for all cases, in both groups, was recorded, including gender, age, and BMI. Intra-operative data was recorded, including pre-operative limb alignment and formal soft tissue release required to achieve coronal balance. Postoperative data was recorded included radiographic limb alignment and component position, specifically distal femoral angle, femoral flexion angle, proximal tibial angle and proximal tibial slope. This data is summarized in Table 1.

A Journey II total knee prosthesis was used for all cases in RA- and Cl-TKA groups.

Post-operative protocols, including enhanced recovery pathways, were identical in both groups studied.

\section{Results}

In the RA-TKA study cohort, a total of 120 patients underwent primary, unilateral TKA during the study period. Similarly, in the control CI-TKA cohort, all patients underwent primary, unilateral TKA. No patients were excluded due to missing data.

Patient demographics are noted in Table 1.

In the RA-TKA group, nine of 120 cases $(7.5 \%)$ required a formal soft tissue release. In the control Cl-TKA group, forty-six of 100 cases (46\%) required a formal soft tissue release.

\section{Discussion/Conclusion}

In this study, patients undergoing both $\mathrm{Cl}$ - and RA-TKA were assessed to evaluate the need for a formal, intra-operative soft tissue release to achieve a "balanced" knee.

While the literature is replete with information about balancing the knee during TKA surgery, a consensus as to what degree of joint laxity should be accepted is not wellestablished. Joint laxity $>3 \mathrm{~mm}$ was chosen prior to the study as the threshold to define whether the soft tissues were balanced or not. This decision was based on previous publications assessing soft tissue laxity in both healthy knees and knees following TKA ${ }^{2,4,8}$. Specifically, data from Heesterbeek et al ${ }^{4}$ demonstrated that soft tissue laxity in knees of healthy, older subjects, at an average age of 62, measured between 2.3 and 3.1 degrees when the medial and lateral soft tissues were stressed with the knee in extension and at 70 degrees of flexion. 
The amount of distraction force necessary to accurately assess soft tissue laxity to define knee balance is also not well-defined. Forces ranging from 15-160 Nm have been reported to be used to various studies ${ }^{4,5}$. In this study, the assessment of soft tissue tensioning was done manually through the use of a posterior cruciate ligament (PCL) Hohmann retractor to consistently apply approximately $120 \mathrm{Nm}$ of force to measure soft tissue laxity.

Overall, 7.5\% of patients undergoing a NAVIO RA-TKA required a formal soft tissue release, compared to $46 \%$ of the $\mathrm{Cl}$-TKA cases. When compared to the control group of $\mathrm{Cl}$-TKA, an $83 \%$ reduction in the need for soft tissue release was documented. Previous studies examining the incidence of formal soft tissue release in $\mathrm{Cl}$-TKA are consistent with these findings, reporting incidences between $50-100 \%{ }^{6}$.

These findings are also consistent with previously reported studies documenting soft tissue releases in 2-15\% of TKA's performed with computer navigation ${ }^{7,8}$.

This study demonstrates that the stated goal of a balanced knee in TKA surgery can be achieved with a high level of precision, without the need for routine soft tissue releases, with the addition of the NAVIO robotic-assisted technology. These findings would suggest that the ability to proactively incorporate gap balancing data, prior to any bony resection, can allow for fine-tuning adjustments to prosthetic size, position and orientation, rather than through the need for reactive soft tissue release techniques, to achieve a wellbalanced TKA. The addition of this robotic-assisted technology in TKA addresses the poor predictive ability of surgeon defined assessment (SDA) of true soft tissue balance in CI-TKA, as documented by MacDessi et $\mathrm{al}^{9}$.

Further study will be necessary to assess whether decreasing the incidence of soft tissue releases will correlate with improved patient-reported outcome measures.

\section{References}

${ }^{1}$ Soft tissue Balancing During Total Knee Arthroplasty in the Varus Knee. Mihalko et al. J Am Acad Orthop Surg 2009;17:766-774.

${ }^{2}$ Accuracy of Soft Tissue Balancing in Total Knee Arthroplasty. Griffin FM, Insall JM, Scuderi G. J Arthroplasty 2000 Dec; 15(8):970-74.

${ }^{3}$ Lessons Learned from Selective Soft-Tissue Release for Gap Balancing in Primary Total Knee Arthroplasty: An Analysis of 1216 Consecutive Total Knee Arthroplasties: AAOS Exhibit Selection. Peters CL et al. J Bone Joint Surg Am 2013 Oct 16;95(20):e152. 
${ }^{4}$ Soft Tissue Tension in Total Knee Arthroplasty. Asano et al. J Arthroplasty 2004 Aug;19(5):558-61

${ }^{5}$ In Vivo Knee Laxity in Flexion and Extension: A Radiographic Study in 30 Older Healthy Subjects. Heesterbeek et al. Knee 2008 Jan; 15(1):45-9

${ }^{6}$ Navigational Predictors in Determining the Necessity for collateral ligament release in Total Knee Replacement. Hakki S et al. J Bone Joint Surg Br. 2009 Sept ; 91-B(9) :1178-1182.

${ }^{7}$ Collateral Soft Tissue Release in Primary Total Knee Arthroplasty. Goudie S, Kamal D. Comput Aided Surg. 2014 Jan; 19(1-3): 29-33.

${ }^{8}$ Navigation as a Predictor of Soft Tissue Release During 90 Cases of Computer-assisted Total Knee Arthroplasty. Saragaglia D et al. Orthop 2006 Oct;29(10):S137-38.

${ }^{9}$ How Accurately Can Soft Tissue Balance Be Determined in Total Knee Arthroplasty? MacDessi SJ et al. J Arthroplasty 2019 Feb; 34(2):P290-294. 


\begin{tabular}{|c|c|c|c|}
\hline Table 1. & & Cl-TKA & $\underline{\text { RA-TKA }}$ \\
\hline BMI & & 31 & 30 \\
\hline Age & (yrs.) & 65 & 70 \\
\hline \multirow[t]{2}{*}{ Gender } & Male (\%) & $44(44 \%)$ & $51(43 \%)$ \\
\hline & Female (\%) & $56(56 \%)$ & 69 (57\%) \\
\hline \multirow[t]{2}{*}{ Preop Extension } & Avg. & $5^{\circ}$ & $1.4^{\circ}$ \\
\hline & Range & $-3-12^{\circ}$ & $-12-16^{\circ}$ \\
\hline \multirow[t]{2}{*}{ Preop Flexion } & Avg. & $116^{\circ}$ & $123^{\circ}$ \\
\hline & Range & $65-130^{\circ}$ & $70-140^{\circ}$ \\
\hline \multirow[t]{2}{*}{ Surgery Time } & Avg. (min) & 53.5 & 56.6 \\
\hline & Range (min) & $38-70$ & $45-75$ \\
\hline \multirow[t]{6}{*}{ Pre-op Alignment } & Varus (\# of cases) & 71 & 98 \\
\hline & Avg. & $4.4^{\circ}$ & $5.2^{\circ}$ \\
\hline & Range & $0-13^{\circ}$ & $0-18^{\circ}$ \\
\hline & Valgus (\# of cases) & 29 & 22 \\
\hline & Avg. & $8.7^{\circ}$ & $4.6^{\circ}$ \\
\hline & Range & $0-20^{\circ}$ & $0-19^{\circ}$ \\
\hline \multirow[t]{2}{*}{ Implant Design } & CR & 74 & 90 \\
\hline & PS & 26 & 30 \\
\hline Post-operative & Distal Femoral Angle & $94.3^{\circ}\left(92-96^{\circ}\right)$ & $93.6^{\circ}\left(92-95^{\circ}\right)$ \\
\hline \multirow[t]{3}{*}{ Radiographic Evaluation } & Femoral Flexion Angle & $1.5^{\circ}\left(0-4^{\circ}\right)$ & $2.3^{\circ}\left(0-5^{\circ}\right)$ \\
\hline & Proximal Tibial Angle & $89.2^{\circ}\left(87-91^{\circ}\right)$ & $89.2^{\circ}\left(86-91^{\circ}\right)$ \\
\hline & Posterior Tibial Slope & $5.3^{\circ}\left(1-7^{\circ}\right)$ & $3.7^{\circ}\left(2-6^{\circ}\right)$ \\
\hline
\end{tabular}

Table 1. Baseline patient demographics and peri-operative data. 